\title{
Avaliação de diferentes substratos na qualidade fisiológica de sementes de melão de caroá [Sicana odorifera (Vell.) Naudim]
}

\author{
LIMA, J.F. ${ }^{1}$; SILVA, M.P.L. ${ }^{2}$; TELES, S. ${ }^{2}$; SILVA, F. ${ }^{2}$; MARTINS, G.N. ${ }^{2}$ \\ 'Centro de Ciências Agrárias, Ambientais e Biológica, Universidade Federal do Recôncavo da Bahia/UFRB, Rua \\ Barreias, 1084, Jardim Cruzeiro, CEP:44040-415, Feira de Santana-Brasil *juliana_firmino@hotmail.com ${ }^{2}$ Centro \\ de Ciências Agrárias, Ambientais e Biológica, Universidade Federal do Recôncavo da Bahia/UFRB, Campus \\ Universitário, s/n, CEP: 44200-000, Cruz das Almas-Brasil
}

\begin{abstract}
RESUMO: O melão de caroá é da família das curcubitáceas encontrada de forma nativa na região do Recôncavo Baiano, onde é muito utilizada pela medicina popular para diversas enfermidades, porém é espécie muito pouco explorada pela pesquisa. O trabalho teve por objetivo estudar diferentes substratos para avaliar o índice de germinação e vigor de sementes de melão de caroá (Sicana odorifera) na produção de mudas. Utilizaram-se como substratos areia lavada, areia lavada com húmus de minhoca (2:1) e substrato comercial Plantmax ${ }^{\circledR}$. Foram realizadas as seguintes avaliações: teste de germinação, índice de velocidade de emergência (IVE), diâmetro de haste, altura de planta, comprimento da raiz, massa seca da raiz, haste e folhas. O substrato comercial Plantmax ${ }^{\circledR}$ promoveu melhor desempenho das sementes em relação aos demais, porém, o mais adequado e de baixo custo para produção de mudas através de sementes de melão de caroá (Sicana odorifera) foi a mistura de areia lavada e húmus de minhoca na proporção 2:1.
\end{abstract}

Palavras-chave: plantas medicinais, geminação, vigor

\begin{abstract}
Evaluation of different substrates in the physiological quality of caroá melon [Sicana odorifera (Vell.) Naudim] seeds. Caroá melon is a curcubitaceae found in its native form in the Recôncavo Region of Bahia, where it is used by popular medicine for many diseases. However, this species is little explored in research. The objective of the present work was to study different substrates in order to evaluate the germination index and seed vigor in caroá melon (Sicana odorifera) during seedling production. The following substrates were used: washed sand, sand washed with worm humus (2:1) and commercial substrate, Plantmax ${ }^{\circledR}$. The following evaluations were carried out: germination test, speed of germination index (SGI), stem diameter, plant height, root length and root, stem and leaf dry matter. The commercial substrate Plantmax ${ }^{\circledast}$ presented the best performance compared to the others; however, the most adequate and least costly in seedling production using caroá melon (Sicana odorífera) seeds, was the mixture of washed sand and worm humus in the 2:1 ratio.
\end{abstract}

Key words: medicinal plants, germination, vigor

\section{INTRODUÇÃO}

O conceito de planta medicinal segundo a OMS é qualquer planta que possui, em um dos órgãos ou em toda planta, substâncias com propriedades terapêuticas ou que sejam ponto de partida na síntese de produtos químicos ou farmacêuticos.

O uso de plantas medicinais pela população mundial tem sido muito significativo nos últimos tempos. Dados da Organização Mundial de Saúde (OMS) mostram que cerca de $80 \%$ da população mundial fez uso de algum tipo de planta na busca de alívio de alguma sintomatologia desagradável. Desse total, pelo menos 30\% deuse por indicação médica. A utilização de plantas medicinais é prática tradicional existente entre os povos de todo o mundo, que tem inclusive incentivos da própria OMS.

O interesse das indústrias farmacêutica, alimentícia e coméstica por produtos originários de plantas medicinais vêm aumentando cada vez mais. Sendo necessária a ampliação de pesquisas que

Recebido para publicação em 05/02/09

Aceito para publicação em 08/12/09

Rev. Bras. PI. Med., Botucatu, v.12, n.2, p.163-167, 2010. 
possa garantir a produção com qualidade da matéria-prima. O número de espécies é muito amplo, porém, os estudos são escassos e as informações vagas. O melão de caroá (Sicana odorífera) é espécie nativa do Recôncavo Baiano pertencente à família das curcubitáceas, onde apresenta potencial a serem utilizados principalmente na indústria farmacêutica.

As curcubitáceas possuem diversos compostos tóxicos ou com potencialidades terapêuticas, como cucurbitacinas, triterpenóides e tetracíclicos oxigenados. De acordo com a farmacopéia, as curcubitáceas exercem varias atividades farmacológicas como antiflamatória, antimicrobiana, antitumoral e muito mais. Conteúdo de tetracíclicos oxigenados se caracteriza também por ter propriedade de produzir espuma em solução aquosa. Também possuem propriedades hemolíticas e, no caso de ser injetada na corrente sangüínea, são muito tóxicas. O melão de caroá também conhecido como melão de caboclo, melão coroa, caju cajuba, cajuá, cagou e cassabanana é utilizada na medicina popular no combate a hipertensão, afecção de pele, anemia, hemorragia, desordem menstrual, doenças do útero, verminose e azia. É composta por ácido ascórbico, água, beta carotenos, cálcio, carboidratos, cinza, ferro, fibra, fósforo, gordura, niacina, proteína, riboflavina e tiamina.

A forma de propagação dessa espécie pode ser tanto por semente quanto por estaquia. Porém, sabe-se que o desencadeamento do processo germinativo de sementes viáveis requer condições ambientais adequadas da umidade, temperatura, oxigênio e luz. O solo deve atender a certos requisitos de textura e estrutura e apresentar boa composição que atenda de forma satisfatória as exigências das sementes.

Substrato é o meio em que as raízes proliferam para fornecer suporte estrutural à parte aérea das mudas e também as necessárias quantidades de água, oxigênio e de nutrientes. Todos os elementos essenciais absorvidos são derivados dos componentes minerais e orgânicos dos substratos Gomes \& Silva (2004). O substrato substitui o solo na produção vegetal. Ele tem a vantagem de poder ser transportado, formulado com matérias primas diversas, ser colocado em recipientes, pode ser manuseado e melhorado. De acordo com Gomes \& Silva (2004), diversos tipos de substratos podem ser utilizados para a produção de mudas e germinação de sementes, dentre eles temos a vermiculita, areia lavada, composto orgânico, moinha de carvão, terra de subsolo, acícula de pinus, estercos, serragem, turfa, bagaço de cana, húmus, além de outros em que o agricultor tenha disponível na propriedade.
O substrato organo-mineral "Plantmax ${ }^{\circledR}$ ", elaborado a base de vermiculita expandida e material orgânico, possui macro e micronutrientes necessários ao desenvolvimento inicial das mudas, boas características físicas, boa capacidade de retenção de água e é livre de pragas e doenças.

O fertilizante orgânico obtido pela decomposição aeróbia controlada produz um composto de boa qualidade, riquíssimo em macro e micronutrientes. Não apresenta acidez e possui elevada taxa de mineralização de N. Todavia devido à alta capacidade de troca catiônica, a liberação de $\mathrm{N}$ é lenta e gradual, reduzindo as perdas por lixiviação. Longo (1992) afirma que o húmus produzido pelas minhocas é em média $70 \%$ mais rico em nutrientes que os húmus convencionais.

Com relação à areia na mistura de substrato, Fachinello et al. (1995) citam que também é material que pode fazer parte do substrato para produção de mudas, por ser de baixo custo, fácil disponibilidade e principalmente por permitir boa drenagem. É substrato que não contem nutrientes e não apresenta propriedades coloidais.

A escolha do substrato é de fundamental importância para desenvolvimento adequado. Para o teste padrão de germinação Brasil (1992) estabelece os substratos papel-toalha e areia.

Os estudos sobre substratos adequados e de baixo custo para produção de mudas de espécies medicinais são escassos. Desta forma, o presente trabalho tem por objetivo estudar diferentes substratos para avaliar a qualidade fisiológica de sementes de melão de caroá (Sicana odorifera).

\section{MATERIAL E MÉTODO}

O trabalho foi conduzido na casa de vegetação de Fisiologia Vegetal do Centro de Ciências Agrárias Ambientais e Biológicas da Universidade Federal do Recôncavo da Bahia (UFRB) que esta localizada na cidade de Cruz das Almas / BA, no período de maio a junho de 2008. A cidade está situada à 1240'19" de Latitude Sul e 3906'22" de Longitude Oeste de Greenwich, tendo $220 \mathrm{~m}$ de altitude. O clima é tropical quente e úmido, Aw a Am, segundo a classificação de Köppen, com temperatura média anual de $24,5^{\circ} \mathrm{C}$ e umidade relativa de $80 \%$ (Almeida, 1999).

As sementes foram extraídas dos frutos em estágio de maturação fisiológica e secas a sombra por 72 horas, em bancada, sobre papel toalha. Em seguida semeadas em bandejas plásticas $(35 \mathrm{~cm}$ de largura interno x $10 \mathrm{~cm}$ de altura) perfuradas no fundo e preenchidas com $2 / 3$ da capacidade volumétrica, com os substratos areia lavada, areia lavada com húmus de minhoca (2:1) e substrato

Rev. Bras. PI. Med., Botucatu, v.12, n.2, p.163-167, 2010. 
comercial Plantmax ${ }^{\circledast}$.

Antes da semeadura cada substrato foi umedecido, a areia foi previamente peneirada e esterilizada em estufa ( $200^{\circ} \mathrm{C}$ por 2 horas), conforme Brasil (1992). A reposição hídrica ocorreu manualmente três vezes ao dia.

As sementes foram submetidas às avaliações de:

Índice de velocidade de emergência

(IVE): realizado através de avaliações diárias, sempre no mesmo horário, a partir do dia em que surgiram as primeiras emergências de plântulas normais. As avaliações foram realizadas até o momento da última contagem e o índice de velocidade foi calculado empregando-se a fórmula de Maguire (1962).

Diâmetro de haste: medido com o auxílio de paquímetro digital na altura do colo da planta e expresso em $\mathrm{cm}$.

Altura da plântula: medida com o auxílio de régua graduada em centímetros do colo ao meristema apical.

Comprimento da raiz principal: medido com o auxílio de régua graduada em centímetros.

A massa da parte aérea, hastes e as raízes das plântulas de cada uma das parcelas foram acondicionadas, separadamente, em sacos de papel e colocadas em estufa com circulação de ar forçado regulada a $80^{\circ} \mathrm{C}$ ate atingir massa constante, efetuando-se as pesagens em balança digital, de três casas decimais, expressos em gramas por plântula.

Os substratos foram analisados quimicamente (Tabela 1). Para a análise da condutividade elétrica, utilizou-se tubetes plásticos submetidos a umedecimento de volume definido por sete dias consecutivos, irrigando com 1/3 do seu volume coletando o lixiviado.

O experimento foi conduzido em delineamento inteiramente casualizado com cinco repetições, o qual cada repetição constou de 50 sementes. Para análise estatística utilizou-se teste $\mathrm{F}$ a $1 \%$ de probabilidade.

\section{RESULTADO E DISCUSSÃO}

O substrato comercial Plantmax ${ }^{\circledR}$ apresentou maior condutividade elétrica (C.E) com base no lixiviado coletado nos primeiros dias de irrigação (Figura 1). Ao final dos sete dias de irrigação a C.E. do lixiviado diminuiu para todos os substratos, com valores nas faixas de 250 a $2000 \mu \mathrm{S} \mathrm{cm}^{-1}$. Silva et al. (2004), trabalhando com mudas de fruteiras encontraram resultado semelhante. A C.E. é proporcional à concentração de nutrientes contidas nos substratos. De acordo com Ehret (1986) citado por Martinez (2004), a condutividade elétrica na ordem de $6 \mathrm{mS} \mathrm{cm}^{-1}$ reduz o tamanho de frutos, a absorção de cálcio, ocasiona toxidez e causa a deficiência de outros íons.

De acordo com a Tabela 2, podemos observar que o substrato comercial Plantmax ${ }^{\circledR}$ apresentou melhor resultado para altura de planta (ALT) e índice de velocidade de emergência (IVE) que os demais substratos testados e comportamento semelhante à mistura de areia lavada mais húmus de minhoca na proporção 2:1 para o comprimento da raiz principal, massa seca de folha (MSF), haste (MSH), raiz (MSR) e total (MST).

Ocorreram diferenças significativas entre os resultados de diâmetro de haste, altura de planta, comprimento de raiz principal, índice de velocidade de germinação, massa seca de folha, massa seca de haste e massa seca da raiz, onde os menores valores foram obtidos com o substrato areia, tornandoo menos indicado para o teste de emergência dessas plântulas, uma vez que não favoreceram o desenvolvimento do sistema radicular das mesmas.

Em sementes de Mimosa regnelli Benthan, a areia foi recomendada para testes de germinação por ter proporcionado boas taxas germinativas, além de ser livre de microrganismos, de baixo custo e reutilizável (Fowler \& Carpanezzi, 1997). Apesar de a areia ser substrato pesado, o que dificulta o manuseio das caixas plásticas no germinador, e exigir reposição de água, mesmo assim ela permitiu boas combinações de germinação e vigor para as sementes de olho-de-

TABELA1. Caracterização química dos substratos avaliados para produção de mudas de melão de caroá (Sicana odorífera) quanto ao $\mathrm{pH} \mathrm{m}$ água, fósforo $(\mathrm{P})$, potássio $(\mathrm{K})$, Cálcio Magnésio $(\mathrm{Ca}+\mathrm{Mg})$, cálcio $(\mathrm{Ca})$, Magnésio $(\mathrm{Mg})$, Alumínio (Al), Enxofre (S), Sódio (Na) e CTC .

\begin{tabular}{l|cccccccccccc}
\hline & \multicolumn{10}{|c}{$\mathrm{mg} \mathrm{dm}^{-3}$} \\
\cline { 2 - 13 } Substratos & $\mathrm{AM}$ & $\mathrm{pH}$ & $\mathrm{K}$ & $\mathrm{Ca}+\mathrm{Mg}$ & $\mathrm{Ca}$ & $\mathrm{Mg}$ & $\mathrm{Al}$ & $\mathrm{H}+\mathrm{Al}$ & $\mathrm{Na}$ & $\mathrm{S}$ & $\mathrm{CTC}$ & $\mathrm{V}$ \\
\hline $\begin{array}{l}\text { Humus + Areia } \\
\text { lavada }\end{array}$ & 794 & 6.20 & 170 & 4,5 & 2,9 & 1,6 & 0,05 & 1,15 & 0,24 & 5,17 & 6,32 & 81,80 \\
Areia lavada & 795 & 5.46 & 40 & 2,3 & 1,6 & 0,7 & 0,05 & 2,47 & 0,10 & 2,55 & 5,02 & 50,79 \\
Plantimax & 796 & 7.90 & 187 & 12,3 & 7,6 & 5,1 & 0,0 & 0,74 & 0,40 & 13,17 & 13,91 & 94,68 \\
\hline
\end{tabular}

Rev. Bras. PI. Med., Botucatu, v.12, n.2, p.163-167, 2010. 


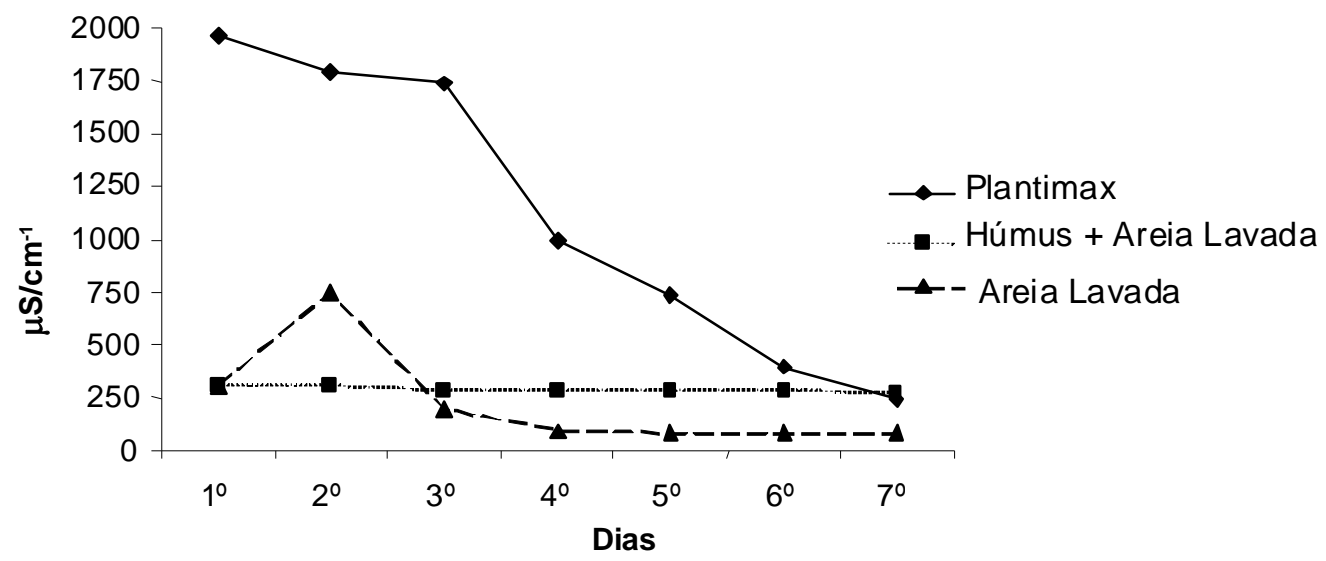

FIGURA 1. Condutividade elétrica dos substratos, Plantmax ${ }^{\circledR}$, húmus + areia lavada (1:2) e areia lavada.

TABELA 2. Média dos tratamentos para as características diâmetro de haste ( $\mathrm{DH}$ - mm), Altura de Planta (ALT $\mathrm{cm})$, Comprimento de raiz principal (CR - $\mathrm{cm}$ ), índice de velocidade de emergência (IVE), massa seca de folha (MSF - g), massa seca de haste (MSH - g), massa seca da raiz (MSR - g) e massa seca total (MST - g) de melão de caroá (Sicana odorifera) cultivados em diferentes substratos em casa - de vegetação no município de Cruz das Almas /BA no mês de junho de 2008

\begin{tabular}{ccccccccc}
\hline & \multicolumn{8}{c}{ Variáveis } \\
\cline { 2 - 8 } Tratamentos & DH & ALT & CR & IVE & MSF & MSH & MSR & MST \\
\hline Areia lavada & $3,2960 \mathrm{~b}$ & $2,6240 \mathrm{c}$ & $11,388 \mathrm{~b}$ & $1,1080 \mathrm{~b}$ & $0,7600 \mathrm{~b}$ & $0,4680 \mathrm{~b}$ & $0,2820 \mathrm{~b}$ & $1,5120 \mathrm{~b}$ \\
$\begin{array}{c}\text { Areia lavada }+ \\
\text { húmus de minhoca } \\
\begin{array}{c}(2: 1) \\
\text { Plantmax }\end{array}\end{array}$ & $3,9960 \mathrm{a}$ & $7,3580 \mathrm{~b}$ & $17,670 \mathrm{a}$ & $1,0620 \mathrm{~b}$ & $2,2160 \mathrm{a}$ & $1,1220 \mathrm{a}$ & $2,4360 \mathrm{a}$ & $5,7700 \mathrm{a}$ \\
\hline Média Geral & $3,7520 \mathrm{a}$ & $8,6520 \mathrm{a}$ & $18,612 \mathrm{a}$ & $1,4340 \mathrm{a}$ & $2,2520 \mathrm{a}$ & $1,1060 \mathrm{a}$ & $1,9380 \mathrm{ab}$ & $5,2980 \mathrm{a}$ \\
\hline
\end{tabular}

Médias seguidas da mesma letra não diferem estatisticamente entre si ao nível de $5 \%$ de probabilidade

pombo. Fanti \& Perez (1999) também constataram que, ao utilizar areia para germinação de sementes de $A$. pavonina, houve dificuldade no que diz respeito à manutenção da umidade, uma vez que houve desuniformidade na capacidade de retenção e distribuição da água. A reposição da umidade deve-se ao fato de este substrato drenar a água, fazendo que a parte superior fique ressecada (Figliolia et al., 1993).

Nunes et al. (2005), observando diferentes substratos na germinação e desenvolvimento de plântulas de moringa obteve melhor resultado com a utilização de areia lavada diferindo com o encontrado para o melão de caroá. Santos et al. (1994) também verificaram, em Mimosa caesalpiniaefolia Benth., que o substrato areia proporcionou os melhores resultados para comprimento de raiz e de parte aérea. Resultados semelhantes foram encontrados por Abreu et al. (2005) e Lopes \& Pereira (2005), trabalhando respectivamente com sementes de Solanum sessiliflorum Dunal e Drimys brasiliensis Miers, que verificaram também, maior porcentagem de germinação das semeadas nos substratos areia, em relação a vermiculita.

Para o cipó-mil-homens (Aristolochia triangularis Cham. Et Schl), Scalon et al. (2007) não encontraram diferença significativa para IVE quando utilizou areia mais terra (1:1), areia mais terra (1:2) e areia, terra mais esterco (1:1:1).

Bezerra et al. (2002) submeteram sementes de Momordica charantia a dois ambientes (temperatura ambiental entre 25-35ㄷ e germinador de sala regulado a $25^{\circ} \mathrm{C}$ ) e quatro substratos (areia, solo, vermiculita e Plugmix) e as sementes responderam positivamente aos substratos areia, solo e plugmix, a germinação baixa foi observada ao tratamento com vermiculita, o Plugmix foi favorável ao desenvolvimento inicial de mudas de Momordica charantia. Já o Plantmax ${ }^{\circledR}$, embora apresente também boas características físicas proporcionou a menor germinação, provavelmente devido a compostos inibidores de germinação presentes na turfa. Por outro lado, a terra de subsolo apresenta baixa porosidade 
e elevada compactação devido ao teor de argila, além de maior possibilidade de contaminações.

Fatores ambientais como a disponibilidade de nutrientes, água, radiação luminosa e a temperatura normalmente têm sido usados para explicar variações intraespecíficas no desenvolvimento das plantas (Wielewicki \& Barros, 2002; Fonseca et al., 2006). Além disto, variações no crescimento do sistema radicular ou aéreo têm sido atribuídas a deficiências nutricionais do solo. De modo geral, o crescimento radicular é favorecido em solos deficientes em nutrientes, notadamente em $\mathrm{N}$ e em P (Marschner et al.,1996), como estratégia para extrair o máximo do nutriente presente no solo (Fernandes, 2000).

Os substratos que proporcionaram maior comprimento das plântulas possivelmente atenderam todos os requisitos para emergência rápida e uniforme, bem como crescimento inicial satisfatório. De acordo com Martins et al. (1999), plântulas que emergem mais rápido podem tornar-se menos vulneráveis as condições adversas do meio por passarem menos tempo nos estádios iniciais de desenvolvimento.

Verificou-se que substrato comercial Plantmax ${ }^{\circledR}$ obteve melhor desempenho em relação aos demais, porém, o mais adequado e de baixo custo para produção de mudas através de sementes de melão de caroá (Sicana odorífera) foi à mistura de areia lavada e húmus de minhoca na proporção 2:1.

\section{REFERÊNCIA}

ABREU, D.C.A.; NOGUEIRA, A.C.; MEDEIROS, A.C.S. Efeito do substrato e da temperatura na germinação de sementes de cataia (Drimys brasiliensis Miers. Winteraceae). Revista Brasileira de Sementes, v.27, n.1, p.149-57, 2005.

ALMEIDA, O.A. Informações meteorológicas do CNP: mandioca e fruticultura tropical. Cruz das Almas: EMBRAPA/CNPMF, 1999. 35p. (EMBRAPA - CNPMF. Documentos, 34).

BELTRÃO, N.E.M. et al. Estimativa da produtividade primária e partição de assimilados na cultura da mamona no semi-árido brasileiro. Revista Brasileira de Oleaginosas e Fibrosas, v.9, n.1/3, p.925-30, 2005. BEZERRA, A.M.E. et al. Germinação e desenvolvimento de plântulas de melão-de-são-caetano em diferentes ambientes e substrato. Ciência Agronômica, v.33, n.1, p.39-44, 2002.

BRASIL. Ministério da Agricultura e Reforma Agrária. Regras para Análise de Sementes. Brasília: LAVARV/ SNAD, 1992. 365p.

FACHINELLO, J.C.; HOFFMANN, A.; NACHTGAL, J.C. Propagação de plantas frutíferas de clima temperado. Pelotas: UFPEL, 1994. 179p.

FANTI, S.C.; PEREZ, S.C.J.G.A. Influência do substrato e do envelhecimento acelerado na germinação de olhode-dragão (Adenanthera pavonina L. - Fabaceae). Revista Brasileira de Sementes, v.21, n.2, p.135-41, 1999.

FERNANDES, L.A. Crescimento inicial, níveis críticos de fósforo e frações fosfatadas em espécies florestais. Pesquisa Agropecuária Brasileira, v.35, p.1191-8, 2000. FIGLIOLIA, M.B.; OLIVEIRA, E.C.; PINÃ-RODRIGUES, F.C.M. Análise de sementes. In: AGUIAR, I.B.; PINÃRODRIGUES, F.C.M.; FIGLIOLIA, M.B. (Eds.). Sementes florestais tropicais. Brasília: Editora, 1993, p.137-74. FONSECA, M.G.; LEÃO, N.V.M.; SANTOS, F.A.M. Germinação de sementes e crescimento inicial de plântulas de Pseudopiptadenia psilostachya (DC.) G. P.Lewis \& M.P.Lima (Leguminosae) em diferentes ambientes de luz. Revista Árvore, v.30, p.120-7, 2006. FOWLER, J.A.P.; CARPANEZZI, A.A. Influência do tipo de substratos e de temperaturas na germinação de sementes de juquiri (Mimosa regnelii Benthan). Colombo: Embrapa-CNPF, 1997. p.1-2. (Comunicado Técnico, 16).

GOMES, M.J.; SILVA, A.R. Os substratos e sua influencia na qualidade de mudas. In: ENCONTRO NACIONAL SOBRE SUBSTRATO PARA PLANTAS. NUTRIÇÃO E ADUBAÇÃO DE PLANTAS CULTIVADAS EM SUBSTRATOS, Anais ... Viçosa, 4., 2004, Viçosa.

LOPES, J.C.; PEREIRA, M.D. Germinação de sementes de cubiu em diferentes substratos e temperaturas. Revista Brasileira de Sementes, v.27, n.2, p.146-50, 2005.

MARSCHNER, H. Mineral nutrient acquisition in nonmycorrhizal and mycorrhizal plants. Phyton- Annales Rei Botanicae, v.36, p.61-8, 1996.

MARTINS, C.C.; NAKAGAWA, J.; BOVI, M.L. Efeito da posição da semente no substrato e no crescimento inicial das plântulas de palmito-vermelho (Euterpe espiritosantensis Fernades - Palmae). Revista Brasileira de Sementes, v.21, n.1, p.164-73, 1999.

MARTINEZ, H.E.P. Distúrbios nutricionais em hortaliças cultivadas em substrato com baixa atividade química. In: BARBOSA, J.G. et al. (Orgs.). Nutrição e adubação de plantas cultivadas em substratos. Viçosa: UFV, 2004. p.129-57.

NUNES, T.A. et al. Efeito do substrato na germinação de sementes e plântulas de Moringa oleifra Lam. Horticultura Brasileira, v.23, n.2, supl., 2005. CD ROM. SANTOS, D.S.B. et al. Efeito do substrato e profundidade de semeadura na emergência e desenvolvimento de plântulas de sabiá. Revista Brasileira de Sementes, v.16, n.1, p.50-3, 1994.

SCALON, S.P. et al. Temperatura, luz e substrato na germinação de sementes de cipó - mil- homens (Aristolochia triangulares Cham. Et Schl). Revista Brasileira de Plantas Medicinais, v.9, n.4, p.32-8, 2007. SILVA, M.P.L. et. al. Substrato para mudas de fruteira a partir de composto de lodo de esgoto. In: NUTRIÇÃO E ADUBAÇÃO DE PLANTAS CULTIVADAS EM SUBSTRATOS. Anais... Viçosa: UFV, 2004. 349p.

WIELEWICKI, A.P.; BARROS, A.C.S. Temperatura e disponibilidade de oxigênio no crescimento de plântulas de arroz irrigado. Revista Brasileira de Sementes, v. 24, p.55-61, 2002. 